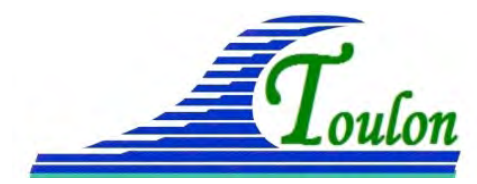

XIV èmes Journées Nationales Génie Côtier - Génie Civil Toulon, 29 juin au $1^{\text {er }}$ juillet 2016

DOI:10.5150/jngcgc.2016.073 C Editions Paralia CFL

disponible en ligne - http://www.paralia.fr - available online

\title{
Exemples de travaux de lutte contre l'érosion hydrologique et marine dans des espaces naturels et urbains littoraux de l'agglomération de Toulon
}

\author{
Claude GUÉRIN ${ }^{1}$ \\ 1 Office National des Forêts (ONF) - Bureau d'Études Méditerranée, \\ 101 Chemin de San Peyre, 83220 Le Pradet, France. \\ claude.guerin@onf.fr
}

\section{Résumé :}

L'érosion hydrologique et marine simultanée est très fréquente sur le littoral varois et aggrave le recul du trait de côte en milieu urbain. Il convient d'apporter un double regard d'hydraulicien et de géotechnicien pour mener à bien des projets de confortement de ce littoral.

Deux exemples de travaux de lutte contre l'érosion hydrologique et marine sont développés ci-après, avec l'accord des collectivités concernées (Département du Var et communauté d'agglomération Toulon Provence Méditerranée (TPM)) :

1) Dans l'Espace Naturel Sensible (ENS) de la Plage du Monaco, commune du Pradet : travaux de maîtrise de l'érosion hydrologique en versant présentant un glissement lent généralisé, couplés à la mise en œuvre d'un gabion bois sur l'escalier d'accès à la plage, protection contre l'érosion marine.

2) Dans la ville de Toulon, sur le tronçon de la plage de la Mitre au Fort Saint Louis : travaux de réparation d'assiette et de confortement de versant aval du sentier du littoral, afin d'assurer une continuité de progression. En effet, l'action érosive des pluies, transitant dans des équipements privés ou publics inadaptés ou trop vétustes, génère des infiltrations et des mouvements de sols diffus. Celle-ci, couplée à l'érosion marine, a provoqué des glissements de terrain sous d'anciennes parades en place ou sur des zones non équipées qu’il convenait de réparer de façon efficace, esthétique et durable.

Mots clés : Érosion, Génie côtier, Environnement littoral.

1. Lutte contre l'érosion hydrologique et marine dans l'ENS de la plage du Monaco. Département du Var - Commune du Pradet

\subsection{Introduction}

L'Espace Naturel Sensible de la plage du Monaco est une ancienne propriété privée sur laquelle les propriétaires avaient pour objectif de construire une maison. Ce projet d'urbanisation a été abandonné, puis cet espace a été cédé au 


\section{Thème 7 - Risques côtiers}

Département du Var. Une piste en versant, bordée de murs de soutènement des terres, et deux escaliers permettent de descendre jusque sur la plage. D'un point de vue géologique, le site est constitué de roches métamorphiques appartenant à la série des phyllades supérieurs de Six-Fours. Elles se caractérisent par une structure en strates et présentent un débit en feuillets leur donnant un aspect friable, naturellement sensible à l'érosion marine et au glissement en période de forte activité hydrologique. Les caractéristiques topographiques et géologiques sont donc défavorables à la stabilité du site : forte pente, circulation d'eau superficielle mal maîtrisée, importante érosion marine, déblais d'anciens travaux déversés dans la pente sans précaution particulière. En 2002, le bas de versant a été partiellement recouvert d'un grillage pendu et en 2008, une partie de ce versant a glissé sur la plage. Le grillage fut alors partiellement arraché mais il limitait la propagation des matériaux sur la plage. De 2009 à 2011, des études géotechniques ont été réalisées sur cet espace et sur la stabilité des trois murs de soutènement de la piste d'accès à la plateforme basse. En 2013, le Département du Var confie à l'Office National des Forêts la maîtrise d'œuvre complète des travaux de restauration d'accès à cet espace et d'amélioration de la protection du versant contre l'érosion. L'enveloppe financière disponible est fixée à $179400 €$ TTC. Le coût des travaux et ingénierie s'élève finalement à $178146 €$ TTC (dont $40427 €$ TTC pour la réalisation du gabion en bois sur la plage).

Maître d’ouvrage : Département du Var

Maître d'œuvre : Office National des Forêts, Bureau d’Études Méditerranée

Entreprises: Synergie Travaux Spéciaux (STS) pour tous les travaux sauf le gabion réalisé en sous-traitance par Attitude Bois Création.

\subsection{Matériel et méthodes}

Deux objectifs sont à satisfaire :

- stabiliser le pied du talus et consolider les escaliers d'accès à la plage,

- stabiliser le versant et les accès par la maîtrise de l'hydrologie superficielle.

Les travaux proposés sont réfléchis eu égard aux contraintes d'accès au site, en maintenant et confortant les dispositifs existants destinés à l'accueil du public.

\subsection{Résultats}

Les escaliers d'accès à la plage sont stabilisés par ancrage de leur pied dans la roche, puis réparés (voir figures 1 et 2).

Concernant les escaliers en versant, ils sont consolidés en remplaçant les emmarchements en bois (voir figure 3). De plus, des renvois d'eau sont ajoutés (voir figure 4) et les cunettes d'évacuation des eaux pluviales sont ancrées en versant. 


\section{XIV èmes Journées Nationales Génie Côtier - Génie Civil \\ Toulon, 29 juin au $1^{\text {er }}$ juillet 2016}

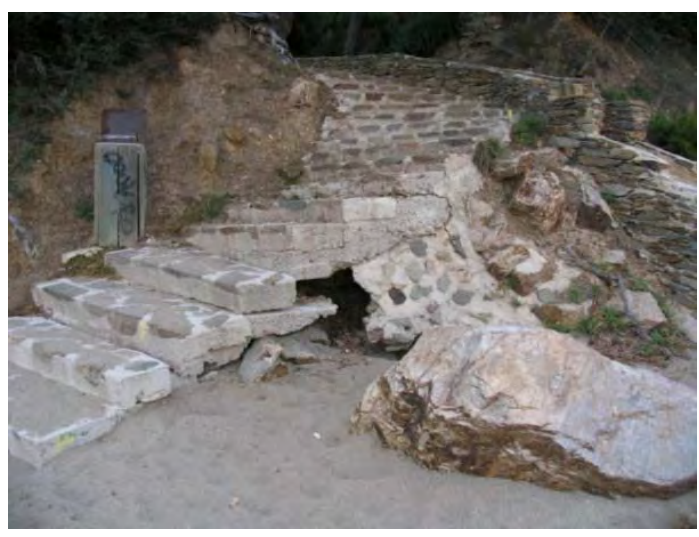

Figure 1. Base de l'escalier Ouest avant travaux.

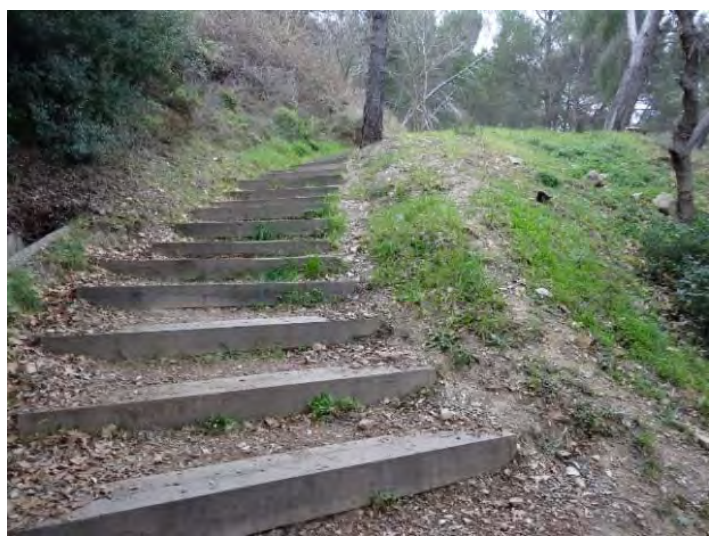

Figure 3. Nouvel emmarchement.

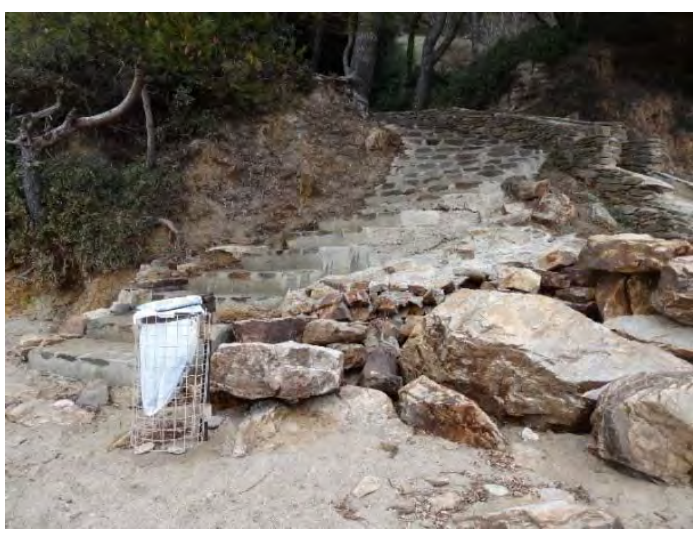

Figure 2. Base de l'escalier Ouest après travaux.

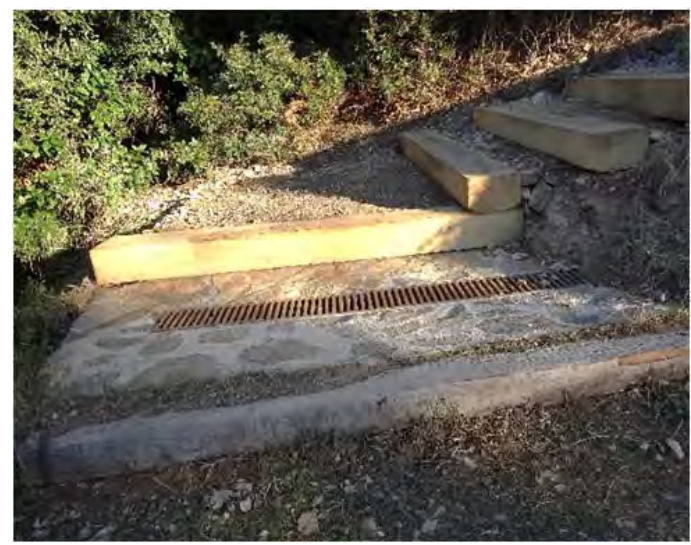

Figure 4. Renvoi d'eau ajouté dans l'emmarchement.

Une surveillance de l'évolution des murs de soutènement, au droit de la piste, est également réalisée en équipant les voiles béton de barrettes ciment témoins, pontant les fissures, afin de déceler un éventuel déplacement différentiel. De 2013 à ce jour, aucun déplacement n’a été mis en évidence par les témoins (voir figures 5 et 6). L’hypothèse du déplacement d'ensemble lent du versant est donc vérifiée. Il est à noter que ce dispositif simple de surveillance a permis de conserver le budget destiné au renforcement de ces murs et de le consacrer aux autres actions liées à la maîtrise de l'hydrologie superficielle.

La collecte des eaux superficielles est améliorée par la mise en place de deux aménagements : une cunette ajoutée d'un renvoi d'eau en thalweg en tête de l'ENS (voir figure 7) et un caniveau à grille en tête de versant bas, afin de limiter les infiltrations (voir figure 8).

Enfin, la stabilisation de l'escalier en pied de versant est réfléchie en recherchant un équipement déformable, léger et transportable par flottaison (voir figure 9). 
Thème 7 - Risques côtiers

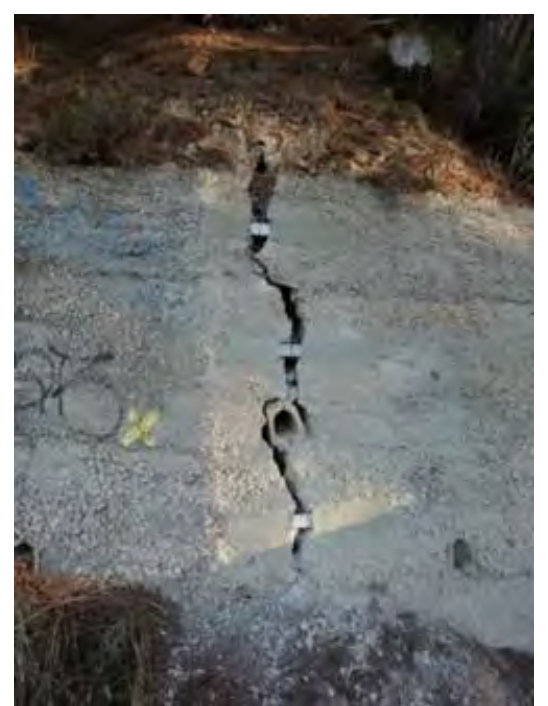

Figure 5. Barre témoin de fissure en 2013.

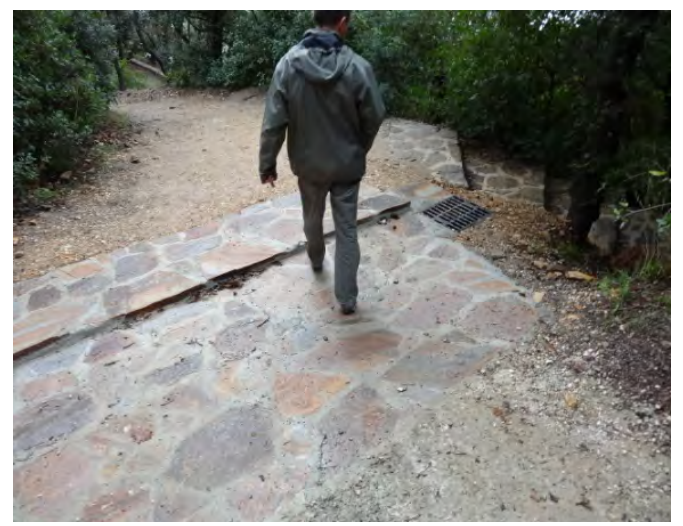

Figure 7. Cunette et renvoi d'eau en amont de l'ENS.

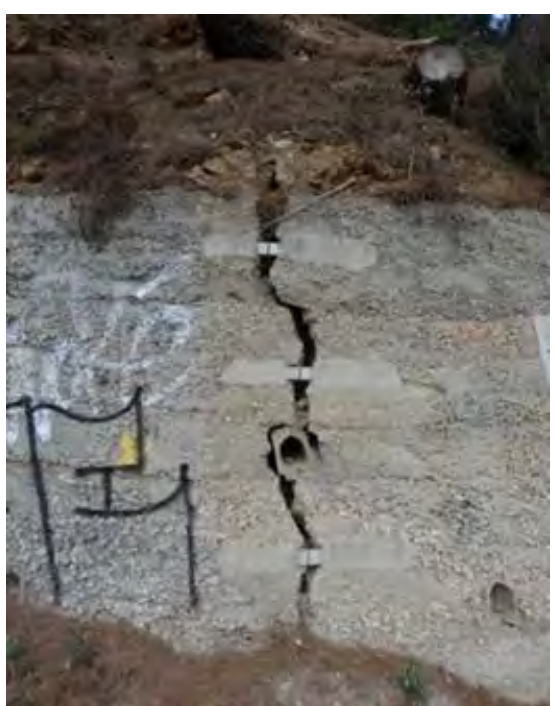

Figure 6. Barre témoin de fissure en 2016.

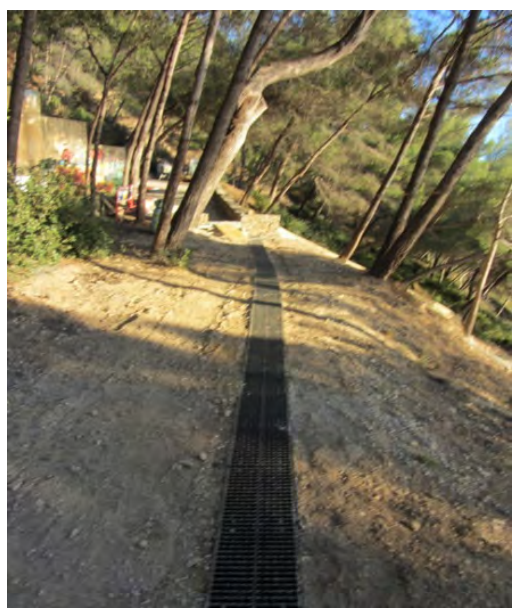

Figure 8. Caniveau sur plateforme amont bas de versant.

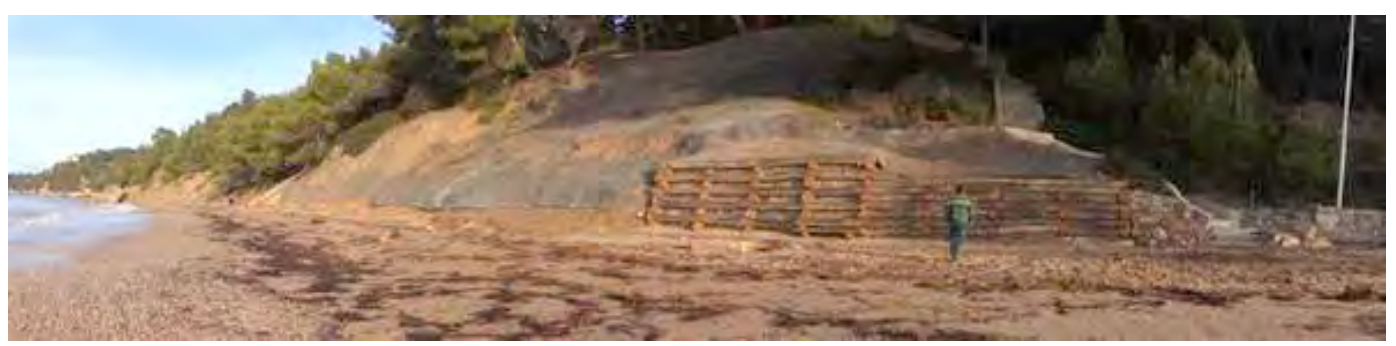

Figure 9. Vue de l'ENS depuis la plage. 


\section{XIV ${ }^{\text {èmes }}$ Journées Nationales Génie Côtier - Génie Civil \\ Toulon, 29 juin au $1^{\text {er }}$ juillet 2016}

Suivant cette optique, le gabion en bois est retenu (voir figures 10 et 11) et mis en place avec les caractéristiques suivantes :

- 18 mètres linéaires montés sur 2,5 m de hauteur et $2 \mathrm{~m}$ de profondeur,

- choix du robinier faux acacia, classe d'environnement 5 "en contact avec la mer",

- écorçage des billes sur la plage (diamètre 30 à $50 \mathrm{~cm}$ sur écorce),

- résidus d'écorçage utilisés en gainage autour des drains dans le corps de l'ouvrage,

- ouvrage fondé à $1 \mathrm{~m}$ sous le niveau bas de la plage, avec un léger pendage amont $\left(10^{\circ}\right)$ sur les phyllades en place,

- deux techniques de confinements des matériaux de remblai sont testées: longrine en bois (est) et pose de pierres plates sur champ (ouest). A ce jour, ces deux techniques possèdent une durabilité équivalente.

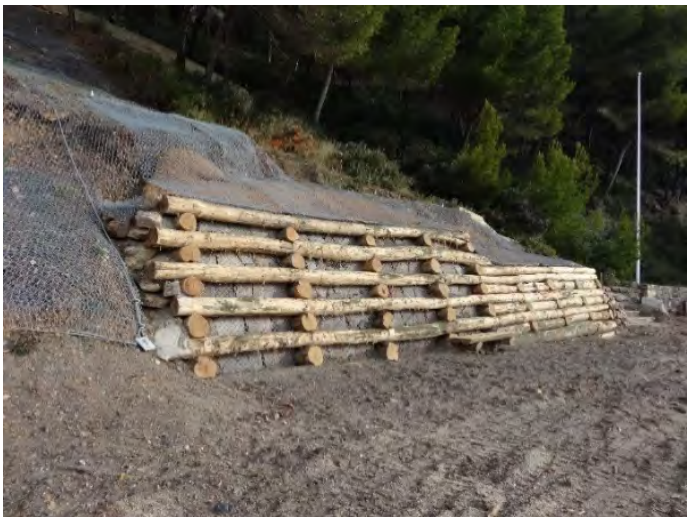

Figure 10. Vue du gabion bois après finition des travaux (2013).

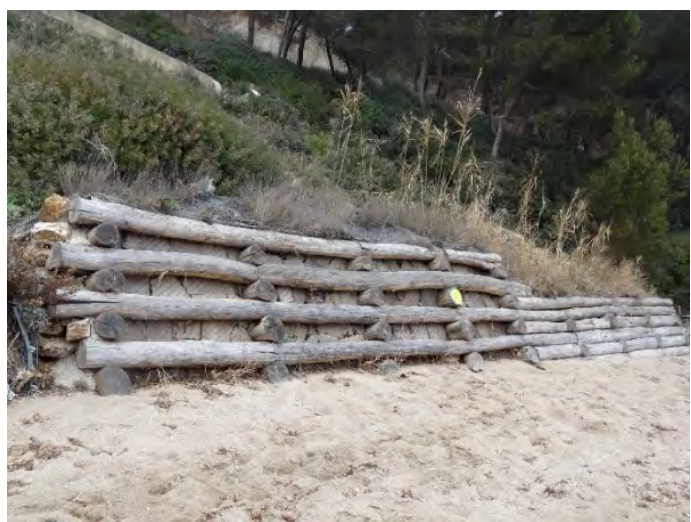

Figure 11. Vue du gabion bois en 2016.

\section{Lutte contre l'érosion hydrologique et marine sur le sentier du littoral, au} lieu-dit La Mitre à Toulon (de la plage de La Mitre au Fort Saint-Louis). Communauté d'agglomération Toulon Provence Méditerranée

\subsection{Introduction}

En 2011, TPM confie à l'ONF la maîtrise d'œuvre pour la réhabilitation et l'entretien du sentier du littoral, sur huit des douze communes constituant la communauté d'agglomération. Ce sentier présente des séquences très diverses et les aménagements doivent être adaptés à l'ambiance de la séquence. Le tronçon de sentier du littoral concerné se situe dans un espace bâti au cœur de la ville de Toulon et est l'objet d'une forte exigence citoyenne (promenade, course à pied, accès aux criques et plages). L'amélioration qualitative recherchée pour les 


\section{Thème 7 - Risques côtiers}

équipements correspond donc à une promenade de bord de mer en station balnéaire (voir figure 12).

D’un point de vue géologique, les sols sont composés de strates diversement décomposées, d'épaisseurs variables et pouvant se superposer, à savoir :

- des terrains superficiels constitués de remblais ou de phyllades très décomprimées,

- des phyllades en place plus ou moins altérées voire fortement altérées.

Au cours de l'hiver 2013/2014, les épisodes pluvieux ont provoqué des écoulements superficiels brutaux et importants, provenant des jardins des propriétés riveraines. Ces eaux se sont concentrées dans des exutoires inappropriés sous les portails d'accès. S'en est suivi une érosion rapide du haut de talus, avec des conséquences importantes sur le sentier du littoral. Des exemples représentatifs du tronçon impacté (zones 6 et 15) sont développés ci-après.

Maître d’ouvrage : Communauté d'agglomération Toulon Provence Méditerranée Maître d'œuvre : Office National des Forêts, Bureau d’Études Méditerranée Entreprises : ALPHAROC/EPC et URBAVAR

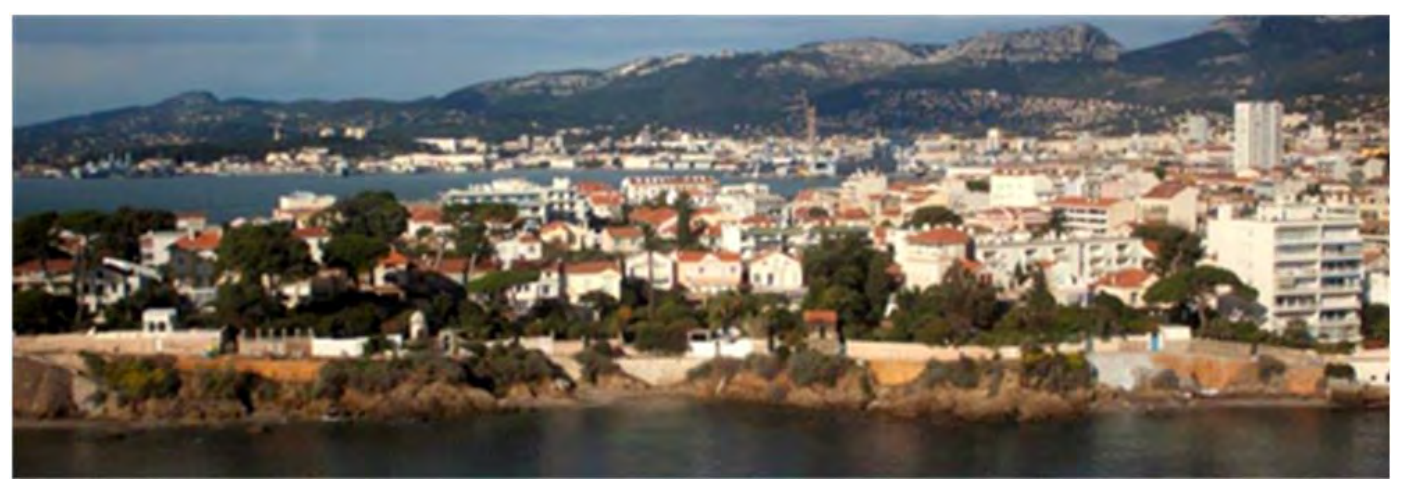

Figure 12. Vue d'hélicoptère du tronçon.

\subsection{Matériel et méthodes}

La portion de sentier concernée par l'étude avait déjà fait l’objet, avant 2011, de réparations sur des glissements ponctuels. En 2013, l'ONF réalise une étude de diagnostic qui met en avant, hormis l'érosion marine de pied, le rôle destructeur des écoulements d'eau superficielle peu maîtrisés (en provenance des jardins à l'aplomb du sentier). L'avant-projet a permis d'affiner les techniques de requalification dans le but d'une meilleure maîtrise de la stabilité et d'un confort accru de l'assiette de cette portion de sentier localisée en zone urbaine très fréquentée. Les travaux consistent finalement en un captage de l'eau superficielle sur différents points du sentier et en son acheminement en aval jusqu’à la roche 


\section{XIV ${ }^{\text {èmes }}$ Journées Nationales Génie Côtier - Génie Civil \\ Toulon, 29 juin au $1^{\text {er }}$ juillet 2016}

dure. Les écoulements en provenance des jardins par canalisation sont vérifiés, ressortis et canalisés en versant aval du sentier.

\subsection{Résultats dans la zone 6}

Début 2014, un ancien glissement est réactivé, il remonte jusqu'au sommet du versant aval et arrache une partie de grillage pendu. Le mur de soutènement de la plateforme du sentier est localement affouillé, l'ouverture d'une faille en bordure de la margelle en béton est observée en tête de talus, ainsi qu'un début de glissement lent des matériaux vers la plage située en contrebas. Lors des fortes pluies, le glissement s'est aggravé par la présence en tête de versant d'une canalisation d'eau pluviale, en provenance du jardin situé en amont du sentier.

Suite à ces observations, deux types de travaux sont réalisés :

- purge du paquet glissé, reprofilage de la zone, démontage de la barrière et pose d'une géogrille naturelle (coco) et d'un grillage ancré haute résistance TECCO sur vingt mètres de longueur et sur les sept premiers mètres de hauteur en tête de versant ; la structure rocheuse étant assez compacte en pied de versant, les trois mètres de hauteur à partir du niveau de la mer ne sont pas équipés car ils sont suffisamment résistants vis-à-vis des coups de mer pour les années à venir (voir figure 13),

- collecte des eaux de ruissellement réalisée par déviation de la canalisation en provenance de la propriété voisine avec regard avaloir, constitution d'une longrine maçonnée faisant office de renvoi d'eau et de support de garde-corps et pavage de la zone pour éviter l'infiltration (voir figure 14).

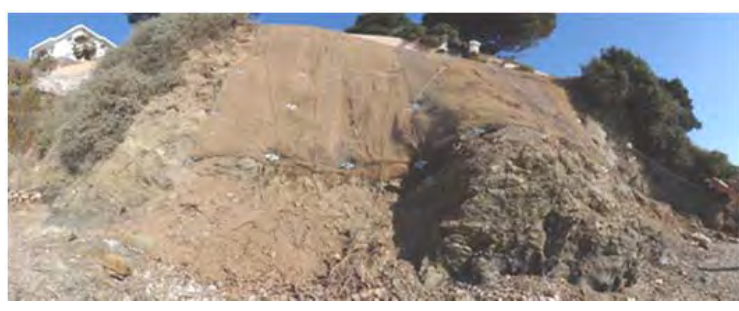

Figure 13. Grillage haute résistance TECCO vu de la plage.

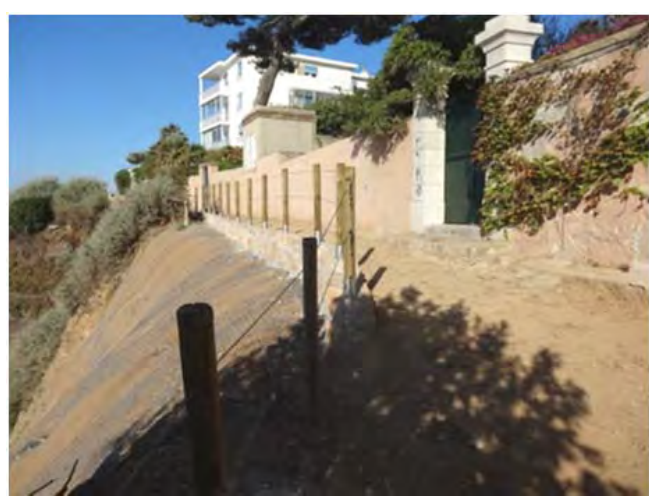

Figure 14. Longrine, garde-corps et pavage vus du sentier.

\subsection{Résultats dans la zone 15}

En septembre 2013, lors de la réalisation de l'étude préliminaire de ce tronçon, le mur maçonné de soutènement a été jugé instable. Un tuyau déversant une eau de qualité douteuse à son aval immédiat avait provoqué un début de glissement de 


\section{Thème 7 - Risques côtiers}

ses fondations ainsi qu'une fissuration de ce mur. Début 2014, lors d'un épisode pluvieux intense, l'affouillement généré par cet écoulement intempestif a provoqué le glissement des fondations et l'écroulement du mur de soutènement de la plateforme, obligeant la fermeture du sentier sur ce tronçon (voir figures 15 et 16).

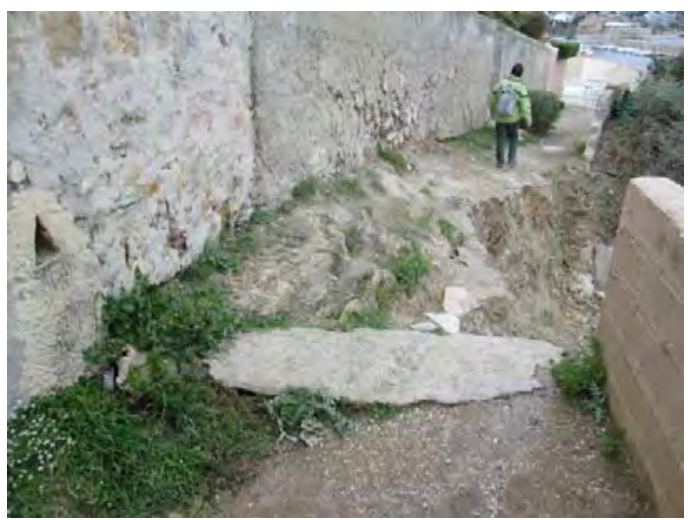

Figure 15. Glissement et écroulement vus du sentier.

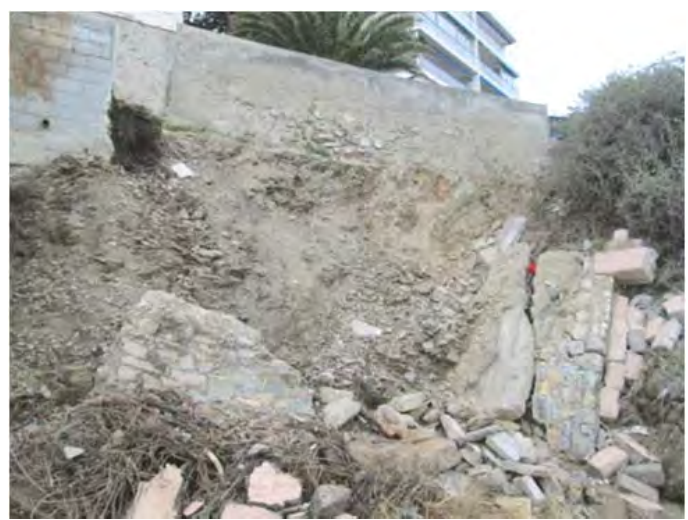

Figure 16. Glissement et écroulement vus de la plage.

Afin de pallier aux dégradations, les travaux suivant sont effectués : déblaiement des parties glissées et reprofilage, mise en œuvre des forages (verticaux pour micropieux, horizontaux pour ancrages), mise en œuvre du coffrage, du ferraillage, des drains subhorizontaux, du tuyau de collecte des eaux de la propriété voisine (voir figure 17), du béton projeté et de la longrine en partie haute, du ballast en remblai et des rambardes. Après test sur la zone, finition de la paroi en béton par un enduit ou par du béton projeté coloré (voir figure 18).

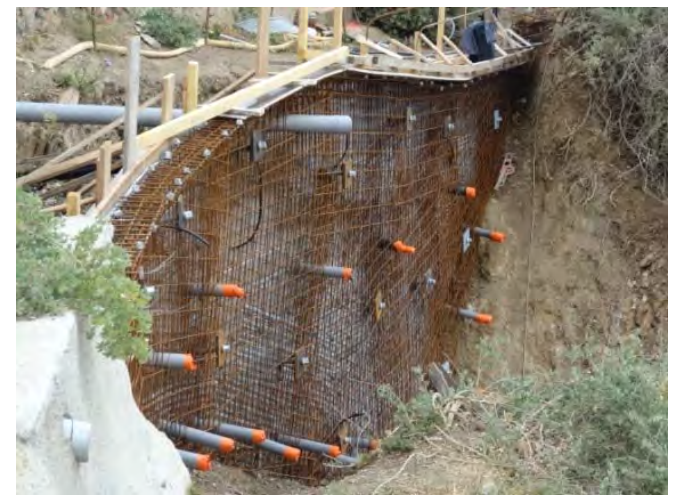

Figure 17. Paroi clouée en cours, drains et tuyau de collecte EP visible.

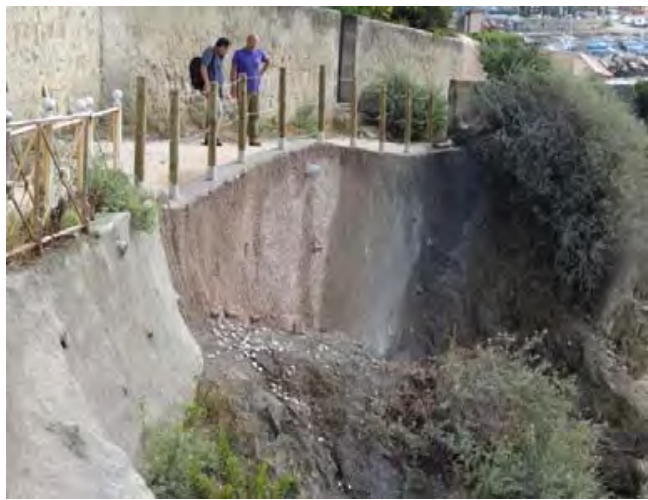

Figure 18. Paroi clouée terminée et test de coloration. 


\section{XIV ${ }^{\text {èmes }}$ Journées Nationales Génie Côtier - Génie Civil \\ Toulon, 29 juin au $1^{\text {er }}$ juillet 2016}

\section{Conclusion}

Dans des zones à enjeu fort, tant en partie naturelle qu'en partie urbaine, la lutte contre l'érosion marine, combinée à une bonne maîtrise des eaux superficielles, freine de façon plus efficace la régression du trait de côte. La réflexion de toute parade à prévoir, pour pallier un phénomène érosif lié à une érosion marine, doit s'accompagner systématiquement d'une analyse de l'hydrologie superficielle ou souterraine à son amont immédiat.

\section{Bibliographie}

ROMAN D. (2009). Guide technique des ouvrages bois dans les cours d'eau, état de l'art, implications et dimensionnement. Rapport ONF, 58 p.

VALLENTIN R. (2011). Etude géotechnique G5 mur de soutènement. Le Pradet (83220), Plage du Monaco. Rapport FONDASOL, 23 p.

VIERLING D. (2010). Réhabilitation et sécurisation de la plage du Monaco. Mise en sécurité, Le Pradet (83). Rapport ERG. 18 p. + annexes.

VIERLING D. (2011). Note de prédimensionnement, phase étude géotechnique de projet. Sentier littoral section La Mitre, mise en sécurité du sentier, Toulon (83). Rapport ERG, 21 p. + annexes.

VIERLING D. (2011). Mise en sécurité du sentier, diagnostic géologique de site. Sentier du littoral, La Mitre, 83000 Toulon. Rapport ERG, 13 p. + annexes. 
Thème 7 - Risques côtiers 